\title{
Complexity in some Physical Systems
}

\author{
R. López-Ruiz \\ Dpto. de Física Teórica, \\ Facultad de Ciencias, Universidad de Zaragoza, \\ 50009-Zaragoza, Spain
}

\begin{abstract}
The LMC-complexity introduced by López-Ruiz, Mancini and Calbet [Phys. Lett. A 209, 321-326 (1995)] is calculated for different physical situations: one instance of classical statistical mechanics, normal and exponential distributions, and a simplified laser model. We stand out the specific value of the population inversion for which the laser presents maximun complexity.
\end{abstract}




\section{Introduction}

The notion of complexity in physics comes from considering the perfect crystal and the isolated ideal gas as examples of simple models, and therefore as systems with zero complexity. The asymptotic properties that an indicator of complexity should have are induced from the characteristics of the two former systems. We have proposed in "López-Ruiz et al., 1995, Calbet \& López-Ruiz, 2001 a simple mathematical expression to calculate this quantity. The result was to define $L M C$-complexity $(C)$ as the interplay between the information $(H)$ stored in a system and its distance to the equipartition, called disequilibrium $(D)$ :

$$
C=H \cdot D=-\left(K \sum_{i=1}^{M} p_{i} \log p_{i}\right) \cdot\left(\sum_{i=1}^{M}\left(p_{i}-\frac{1}{M}\right)^{2}\right),
$$

where $\left\{p_{1}, p_{2}, \ldots, p_{M}\right\}$ represent the probabilities of the $M$ states $\left\{x_{1}, x_{2}, \ldots, x_{M}\right\}$

accessible to the system, with the normalization condition $\sum_{i=1}^{N} p_{i}=1 ; H$ gives the Shannon information, where $K$ is a constant [Shannon \& Weaver, 1949] and $D$ is a measure of a probabilistic hierarchy among the states. This definition presents advantages since it is based upon a probabilistic description of the system, and the knowledge of the probability distribution of its accesible states allows to calculate the LMC-complexity. The quantity $C$ is associated to a particular description and therefore to a scale of observation, just as intuition suggests.

In the following sections we calculate $C$ in different physical situa- 
tions. Sections 2. and 3. are devoted to obtain LMC-complexity for a system in thermal equilibrium and for the normal and the exponential probability distributions. In Section 4. the previous results are applied to study the behavior of $C$ in a simplified model of a two-level laser system.

\section{Complexity in the Canonical Ensemble}

Each physical situation is closely related to a specific distribution of microscopic states. Thus, an isolated system presents equipartition, by hypothesis: the microstates compatible with a macroscopic situation are equiprobable [Huang, 1987]. The system is said to be in equilibrium. For a system surrounded by a heat reservoir the probability of the microstates associated to the thermal equilibrium follow the Boltzmann distribution. In general, the stablished scheme consists in associating a probability distribution of states to each phenomenon. If the system presents some specific distribution it is said to be in some kind of equilibrium. From this point of view, complexity $C$ can be assigned to each system depending on the specific description.

Let us try to analyse the behavior of $C$ in an ideal gas in thermal equilibrium. In this case the probability $p_{i}$ of each accesible state is 
given by the Boltzmann distribution:

$$
\begin{aligned}
p_{i} & =\frac{e^{-\beta E_{i}}}{Q_{N}}, \\
Q_{N} & =\int e^{-\beta E(p, q)} \frac{d^{3 N} p d^{3 N} q}{N ! h^{3 N}}=e^{-\beta A(V, T)},
\end{aligned}
$$

where $Q_{N}$ is the partition function of the canonical ensemble, $\beta=1 / \kappa T$ with $\kappa$ the Boltzmann constant and $T$ the temperature, $V$ the volume, $\mathrm{N}$ the number of particles, $E(p, q)$ the hamiltonian of the system, $h$ is the Planck constant and $A(V, T)$ the Helmholtz potential.

Calculation of $H$ and $D$ gives us:

$$
\begin{aligned}
H(V, T) & =\left(1+T \frac{\partial}{\partial T}\right)\left(\kappa \log Q_{N}\right)=S(V, T), \\
D(V, T) & =e^{2 \beta[A(V, T)-A(V, T / 2)]} .
\end{aligned}
$$

Note that Shannon information $H$ coincides with the thermodynamic entropy $S$ when $K$ is identified with $\kappa$. If a system verifies the relation $U=C_{v} T$ ( $U$ the internal energy, $C_{v}$ the specific heat) the complexity takes the form:

$$
C(V, T) \sim \operatorname{cte}(V) \cdot S(V, T) e^{-S(V, T) / \kappa}
$$

that matches the intuitive function proposed in Figure 1. 


\section{Complexity in Distributions}

The introduced indicator, LMC-complexity, is closely related to the probability distribution of states associated to a system. We remark this idea calculating $C$ for the normal and exponential distributions.

Normal Distribution: Suppose a continuum of states represented by the $x$ variable whose probability density $p(x)$ is given by the normal distribution of variance $\sigma$ :

$$
p(x)=\frac{1}{\sigma \sqrt{2 \pi}} \exp \left(-\frac{x^{2}}{2 \sigma^{2}}\right) .
$$

The expressions obtained for $H, D$ and $C$ are the following:

$$
\begin{aligned}
H & =-K \int_{-\infty}^{+\infty} p(x) \log p(x)=K\left(\frac{1}{2}+\log (\sigma \sqrt{2 \pi})\right) \\
D & =\int_{-\infty}^{+\infty} p^{2}(x) d x,=\frac{1}{2 \sigma \sqrt{\pi}} \\
C_{g} & =H \cdot D=\frac{K}{2 \sigma \sqrt{\pi}}\left(\frac{1}{2}+\log (\sigma \sqrt{2 \pi})\right) .
\end{aligned}
$$

The additional condition $H \geq 0$ imposes $\sigma \geq \sigma_{\min }=(2 \pi e)^{-1 / 2}$. The highest complexity is reached for a determined width: $\bar{\sigma}=\sqrt{(e / 2 \pi)}$.

Exponencial Distribution: Consider a exponencial distribution of 
variance $\gamma$ :

$$
p(x)= \begin{cases}\frac{1}{\gamma} e^{-x / \gamma} & x>0 \\ 0 & x<0 .\end{cases}
$$

The same calculation gives us:

$$
\begin{aligned}
H & =K(1+\log \gamma) \\
D & =1 / 2 \gamma \\
C_{e} & =\frac{K}{2 \gamma}(1+\log \gamma)
\end{aligned}
$$

with the condition $\gamma \geq \gamma_{\min }=e^{-1}$. The highest complexity corresponds in this case to $\bar{\gamma}=1$.

In Fig. 2. the dependence of $C$ on width $(\sigma=\gamma)$ is represented. Remark that for the same width the exponential distribution presents a higher complexity $\left(C_{e} / C_{g} \sim 1.4\right)$.

\section{Complexity in a Two-Level Laser Model}

One step further, combining the results obtained in Secs. 2. and 3., is now done. We calculate LMC-complexity for an unrealistic and simplified model of laser Svelto, 1989.

Let us suppose a laser of two levels of energy: $E_{1}=0$ and $E_{2}=\epsilon$, with $N_{1}$ atoms in the first level and $N_{2}$ atoms in the second level, and the 
condition $N_{1}+N_{2}=N$ (the total number of atoms) (Fig. 3.). Our aim is to sketch the statistics of this model and to introduce the results of photon counting Arecchi, 1965 that produces an asymmetric behavior of $C$ as function of the population inversion $\eta=N_{2} / N$. In the range $\eta \in(0,1 / 2)$ spontaneous and stimulated emission can take place, but only in the range $\eta \in(1 / 2,1)$ the condition to have lasing action is reached, because the population must be, at least, inverted, $\eta>1 / 2$.

The entropy $(S)$ of this system vanishes when $N_{1}$ or $N_{2}$ is zero. Moreover, $S$ must be homegenous of first order in the extensive variable $N$ Callen, 1985. For the sake of simplicity we approach $S$ by the first term in the Taylor expansion:

$$
S \sim \kappa \frac{N_{1} N_{2}}{N}=\kappa N \eta(1-\eta)
$$

The internal energy is $U=N_{2} \epsilon=\epsilon N \eta$ and the statistical temperature is:

$$
T=\left(\frac{\partial S}{\partial U}\right)_{N}^{-1}=\frac{\epsilon}{\kappa} \frac{1}{(1-2 \eta)} .
$$

Note that for $\eta>1 / 2$ the temperature is negative as corresponds to the stimulated emission regime dominating the actual laser action.

From Eq. (5) the value of disequilibrium is in this case:

$$
D(N, T)=e^{N / 4} e^{-2 S / \kappa}
$$

and then LMC-complexity is:

$$
C=e^{N / 4} \cdot S e^{-2 S / \kappa}
$$


In the laser regime, the quantity $C$ can reach the same order of magnitude of $S$ when $D \sim 1$.

We are now interested in introducing qualitatively the results of laser photon counting in the calculation of LMC-complexity. It was reported in Arecchi, 1965 that the photo-electron distribution of laser field appears to be poissonian. In the continuous limit the Poisson distribution is approached by the normal distribution Harris \& Stocker, 1998]. The width $(\sigma)$ of this energy distribution in the canonical ensemble is proportional to the statistical temperature of the system. Thus, for a switched on laser in the regime $\eta \in[1 / 2,1]$, the width of the gaussian energy distribution can be fitted by choosing $\sigma \sim-T \sim 1 /(2 \eta-1)$ (recall that $T<0$ in this case). The range of variation of $\sigma$ is $\left[\sigma_{\infty}, \sigma_{\min }\right]=\left[\infty,(2 \pi e)^{-1 / 2}\right]$. Then we obtain:

$$
\sigma \sim \frac{(2 \pi e)^{-1 / 2}}{2 \eta-1}
$$

By replacing this expression in Eq. (8), and rescaling by a factor proportional to entropy, $S \sim \kappa N$, (in order to give to it the correct order of magnitude), LMC-complexity for a population inversion in the range $\eta \in[1 / 2,1]$ is reobtained:

$$
C_{\text {laser }} \simeq \kappa N \cdot(1-2 \eta) \log (2 \eta-1) .
$$

We consider at this level of discussion $C_{\text {laser }}=0$ for $\eta<1 / 2$. The behavior of this function for the whole range of parameter $\eta \in[0,1]$, is plotted in Fig. 4. It is worth noticing the value $\eta_{2} \simeq 0.68$ where the laser 
presents the highest complexity.

By following theses ideas, if the width, $\sigma$, of the experimental photoelectron distribution of laser field is measured, the population inversion parameter, $\eta$, would be given by Eq. (15). In a next step, we would obtain the LMC-complexity of the laser system by Eq. (16).

\section{Conclusions}

A model helps us to approach the reality and provides invaluable guidance in the objetive of a finer understanding of a physical phenomenon. From this point of view the present work tries to enlighten the problem of calculating the $L M C$-complexity, $C$, of a physical system via a simplified model. Thus, an unrealistic presentation of a two-level laser system has been worked out. In this context, we have obtained an expression for the quantity $C$ as a function of the population inversion, $\eta \in[0,1]$. The laser presents the highest complexity for $\eta \simeq 0.68$. A formal experimental approach to its measurement, if possible, is proposed.

Acknowledgements The author wants to thank Prof. H.L. Mancini, from Universidad Privada de Navarra (Spain), for very useful and fruitful discussions. 


\section{References}

[López-Ruiz et al., 1995]

López-Ruiz, R., Mancini, H.L. \& Calbet, X. [1995], A statistical measure of complexity, Phys. Lett. A 209, 321-326.

[Calbet \& López-Ruiz, 2001]

Calbet, X. \& López-Ruiz, R. [2001], Tendency to maximum complexity in a non-equilibrium isolated system, Phys. Rev. E 63, 066116(9).

[Shannon \& Weaver, 1949]

Shannon, C.E. \& Weaver, W. [1949], The Mathematical Theory of Communication (University of Illinois Press, Urbana).

[Huang, 1987]

Huang, K. [1987], Statistical Mechanics (John Wiley \& Sons, New York).

[Svelto, 1989]

Svelto, O. [1989], Principles of Lasers (Plenum Press, New York).

[Arecchi, 1965]

Arecchi, F.T. [1965], Measurement of the statistical distribution of gaussian and laser sources, Phys. Rev. Lett. 15, 912-916. 
[Callen, 1985]

Callen, H.B. [1985], Thermodynamics and an Introduction to Thermostatistics (J. Whiley \& Sons, New York).

[Harris \& Stocker, 1998]

Harris, J.W. \& Stocker, H. [1998], Handbook of Mathematics and Computational Science (Springer-Verlag, New York). 


\section{Figure Captions}

Fig 1. Sketch of the intuitive notion for the magnitudes: information $(\mathrm{H})$, disequilibrium (D) and complexity for the physical systems. Extreme systems are the perfect crystal and the isolated ideal gas.

Fig 2. LMC-Complexity $(C=H \cdot D)$ as a function of the width $(\sigma=\gamma)$ for the normal and exponential distributions. (It is suppossed $K=1)$.

Fig 3. Diagram showing the two-level laser model used in Sec. 4.

Fig 4. LMC-Complexity on the population inversion $(\eta)$ for the twolevel laser model. Observe the peak in complexity for $\eta_{2} \simeq 0.68$ (lasing regime). (Units in $C$ scaled by $\kappa N$ ). 